\title{
„Iron Heart": Reversible Cause of Dilated Cardiomyopathy Secondary to Cardiac Toxicity in Elderly Patient with Myelodysplastic Syndrome
}

Ana Martin-Garcia', Marta Alonso Fernandez de Gatta', María Diez-Campelo², Agustín C. Martín-García ${ }^{1}$, Manuel Barreiro Pérez ${ }^{1}$, Elena Diaz-Pelaez', Félix López Cadenas ${ }^{2}$, Pedro L. Sanchez ${ }^{1}$

${ }^{1}$ Cardiology Department, University Hospital of Salamanca-IBSAL-CIBERCV

${ }^{2}$ Hematology Department, University Hospital of Salamanca-IBSAL

Correspondence:

Ana Martin-Garcia

Cardiology Department, University Hospital of Salamanca-IBSAL-CIBERCV

58-182 Paseo de San Vicente 37007 Salamanca, Spain e-mail: anamartin.amg@gmail.com phone: 0034-630703819 fax: $+34-923-270008$

Received:

3.07.2019

Accepted:

21.09.2019

DOI: 10.24292/01.OR.319210919

Copyright $\odot$ Medical Education. All rights reserved.

\section{ABSTRACT}

The most common non-hematological cause of death in patients with low-risk myelodysplastic syndrome (MDS) who receive chronic supportive transfusion therapy (STT) through red blood cells (RBC) is known to be related to cardiac events, especially, cardiac siderosis [1].

At the time patients develop symptoms secondary to cardiac toxicity, the disease reaches an advanced phase with a very high mortality rate. However, we report the case of an old female patient with severe acute heart failure (HF) and signs of elevated myocardial iron deposits by cardiac magnetic resonance, who experienced an unexpected and marked clinical improvement using a combination of intensive iron chelation therapy (ICT) and specific treatment for HF. She overcame the HF acute phase, recovered her systolic function and remained stable at mid-term of follow-up (18 months).

Key words: myelodysplastic syndrome, supportive transfusion therapy, cardiotoxicity, cardiac magnetic resonance, intensive iron chelation therapy 


\section{CASE REPORT}

We present a 77-year-old lady diagnosed with myelodysplastic syndrome (MDS) type refractory anemia with ring sideroblasts (RARS) since 2006 and under every two weeks supportive transfusion therapy (STT) (191 packed red blood cells [RBC]). She suffered intolerance to iron chelation (deferoxamine) and history of erythropoietin therapy fail in the past. She was referred to our Emergency Department in September 2014 presenting with cardiogenic shock (acute heart failure). Transthoracic echocardiogram showed severe biventricular dilatation and systolic dysfunction (video), severe mitral and tricuspid regurgitation, restrictive mitral inflow pattern and high suspicion of pulmonary hypertension. The patient was admitted to our Acute Cardiovascular Care Unit. Intensive medical treatment was required using inotropic and vasopressor drugs. Blood tests reflected a hemoglobin level of $8.5 \mathrm{~g} / \mathrm{dL}$, increased of precursor of brain natriuretic peptide (NT-BNP, $9854 \mathrm{pg} / \mathrm{mL}$ ) and abnormal iron metabolism parameters (ferritin $-6180 \mathrm{~g} / \mathrm{L}$-, and elevated transferrin saturation index $-106.1 \%$-). A coronary angiography revealed no obstructive coronary artery disease.

Accordingly, a cardiac magnetic resonance (CMR) was performed to rule out secondary hemochromatosis (video). It showed dilatation and biventricular systolic dysfunction (LV volumen: $129 \mathrm{ml} / \mathrm{m}^{2}$ [N $<84 \mathrm{ml} / \mathrm{m}^{2}$ ], LVEF 28\%), absence of late gadolinium enhancement and signs of elevated myocardial iron deposits with a significant reduction in $\mathrm{T} 2 \mathrm{star}(\mathrm{T} 2 *)$ relaxation time in the middle ventricular septum $(7.6 \mathrm{~ms}, \mathrm{~N}>20 \mathrm{~ms})$. These findings confirmed the diagnosis of cardiac hemosiderosis. Moreover, $\mathrm{T}^{*}$ time in liver corroborated concomitant severe hepatic overload (fig. 1).

FIGURE 1.

Cardiac magnetic resonance revealed significant myocardial and hepatic iron deposits. The use of $\mathrm{T} 2 *$ mapping allowed to quantify iron deposits in both the myocardium and the liver.

\section{T2* mapping at admission (September 2014)}

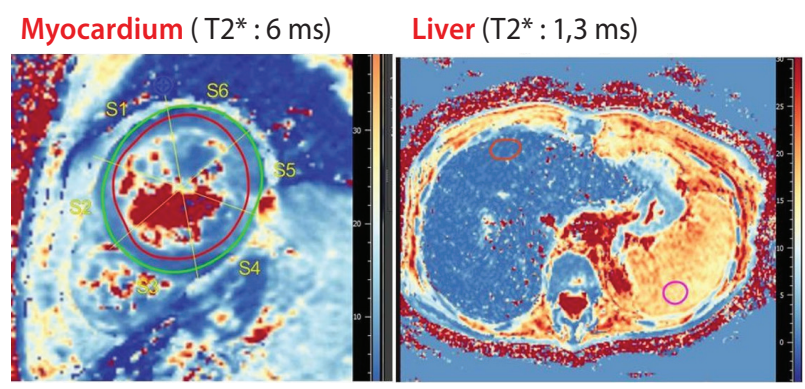

Following the indications of our Hematology Department, intensive iron chelation therapy was initiated by combination of intravenous deferoxamine (50 mg/kg/day) and oral deferiprone (1000 mg/8 hours). This combined therapy is not available for routine use in our hospital so it was requested as a compassionate treatment and the patient signed a detailed informed consent.

The patient improved her general condition during the admission and fortunately, she was discharged keeping on treatment with deferiprone $1000 \mathrm{mg}$ td orally and standard HF treatment according to current Clinical Practice Guidelines for HF. Hence, the patient was followed in the outpatient clinic and optimal dose titration was progressively achieved.

Ecocardiography and cMR were repeated at 3,12 and 18 months from admission. In the last clinic during the follow-up, she referred a good functional class (NYHA class I). Furthermore, the biventricular systolic function was normalized (fig. 2, video) and NT-proBNP value had significantly decreased $(<800 \mathrm{pg} / \mathrm{ml})$. In August 2016, she died of non-cardiologic cause, due to an accidental fall with traumatic brain injury and extensive parenchymal hematoma on a cerebral CT scan.

\section{DISCUSSION}

Cardiac siderosis related to STT in young adults with thalassemia major (TM) is associated with a poor cardiovascular prognosis. However, it has been described that it might be treated in early stages with iron chelation therapy with a higher probability of success and safer method compared to advanced phases. Patients with TM who develop acute HF had a high in-hospital mortality which reaches up to $50 \%$ and their mean survival time in complex cases with extensive cardiac involvement may be less than one year [2].

Although in patients with MDS, cardiac complications are the most common cause of death not related to the hematological process [1], there is little data regarding the diagnosis and treatment of cardiac siderosis, being considered as an irreversible disease. In contrast, recently, some authors have shown that the use of iron chelators significantly decreases the occurrence of cardiac events in these complex patients and also delays their development [3].

The relevance of the case we present is to be the first published in the scientific literature, in which an elderly female patient with SMD and acute heart failure (HF) in the context of dilated cardiomyopathy secondary to cardiac toxicity with severe biventricular systolic dysfunction, overcame the HF acute phase, recovered her systolic function with intensive combined iron chelation and 


\section{FIGURE 2.}

Temporal evolution of the findings obtained by cardiac magnetic resonance. Cardiac magnetic resonance showed a significant and progressive improvement of LVEF and RVEF, a reduction of left ventricular end-diastolic volume and a discrete increase in $\mathrm{T} 2$ star relaxation time.

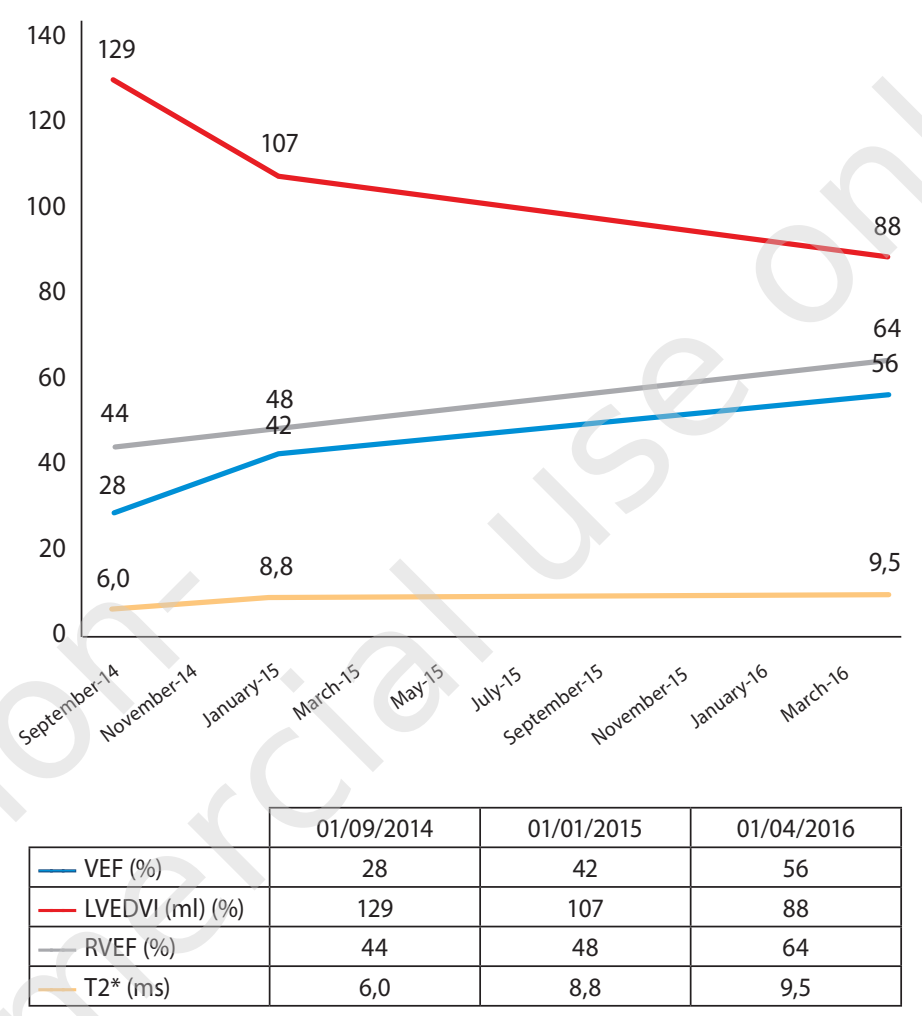

CMR - cardiac magnetic resonance; LVEF - left ventricle ejection fraction; RVEF - right ventricle ejection fraction; LVEDVI - left ventricle end-diastolic volume index.

VIDEO.

Transthoracic echocardiography and cardiac magnetic resonance performed on admission and follow up. Admission TTE and cMR showed severe biventricular dilatation and systolic dysfunction with severe mitral regurgitation. TTE and cMR at 18 months after admission, revealed the recovery of biventricular systolic function with intensive combined iron chelation and medical treatment for heart failure.

TTE - transthoracic echocardiography; cMR - cardiac magnetic resonance

Additional material (video) available on the website: www.oncoreview.pl. http://mededu.home.pl/movie/video.mov

medical treatment for $\mathrm{HF}$, and remained stable at mid-term of follow-up (18 months).

Nowadays, the CMR is well-established as the gold standard method for the quantification of myocardial iron deposition [4]. This technique obtains a quantitative parameter, $\mathrm{T} 2 *$ relaxation time, which is defined as the rate of signal loss in tissues with pathological iron deposit due to loss of homogeneity of the magnetic field caused by hemosiderin: the increase of the deposit leads to a decrease of T2* time [5]. T2* has been reported to be the most reliable, reproducible and clinically viable method for quantifying cardiac iron overload [5].

Values of $\mathrm{T}^{*}>20 \mathrm{~ms}$ reflect the absence of pathological myocardial iron deposition, while $\mathrm{T}^{*}<6 \mathrm{~ms}$ indicate severe myocardial siderosis with a $50 \%$ risk of developing cardiac dysfunction in a year [6]. Nevertheless, this technique is not routinely performed due to its cost and the lack of availability in most of the hospitals. 


\section{SUMMARY}

Due to the significant clinical and therapeutic consequences of the detection of pathologic deposits of myocardial iron and the potential reversibility of this cardiac toxicity reflected in our case, we might consider that patients undergoing chronic STT should be exhaustively evaluated through the most current accurate techniques to identify and quantify myocardial iron as well as the morphological and functional consequences of cardiac siderosis, in order to initiate the appropriate medical treatment.

\section{SOURCES OF FOUNDING}

This work is funded in part by Instituto de Salud Carlos III (European Regional Development Fund, ERDF, PIE14/00066) and the Gerencia Regional de Salud de Castilla y León (GRS 1203/A/15).

\section{References}

1. Dayyani F, Conley AP, Strom SS et al. Cause of death in patients with lower-risk myelodysplastic syndrome. Cancer 2010; $116: 2174-9$.

2. Gujja P, Rosing DR, Tripodi DJ, Shizukuda Y. Iron overload cardiomyopathy: better understanding of an increasing disorder. J Am Coll Cardiol 2010; 56:1001-12.

3. Remacha AF, Arrizabalaga B, Villegas A et al. Evolution of iron overload in patients with low-risk myelodysplastic syndrome: iron chelation therapy and organ complications. Ann Hematol 2015; 94: 779-87.

4. Modell B, Khan M, Darlison M et al. Improved survival of thalassaemia major in the UK and relation to T2* cardiovascular magnetic resonance. J Cardiovasc Magn Reson 2008; 10: 42.

5. Tanner MA, He T, Westwood MA et al. Multi-center validation of the transferability of the magnetic resonance T2* technique for the quantification of tissue iron. Haematologica 2006; 91: 1388-91.

6. Wood JC. Impact of iron assessment by MRI. Hematology Am Soc Hematol Educ Program 2011; 2011: 443-50.

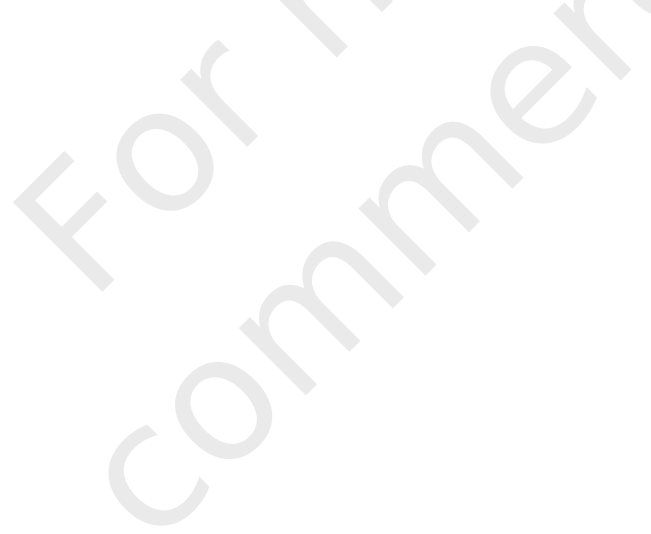

Authors' contributions:

All authors had made an individual contribution to the writing of the article and not just been involved with the patient's care. All authors had made substantial contributions to the following: conception and design, acquisition of data or analysis and interpretation of data, drafting the article or revising it critically for important intellectual content, final approval of the version published.

Conflict of interests: None.

Financial support: 